\title{
Gene Expression Analysis in Rat Pancreas Observed with Whole-Transcript Exon Array after Ventromedial Hypothalamic Lesions
}

\author{
Takayoshi Kiba \\ Division of Modern Medical Technology, Institute for Clinical Research, National Hospital Organization \\ Kure Medical Center and Chugoku Cancer Center, Kure, Japan
}

\section{Keywords}

Ventromedial hypothalamic lesions - Gene expression profiling · Pancreas · Rat · Whole-transcript exon array

\begin{abstract}
Background: It was reported previously that using Affymetrix Rat Genome 230 2.0, one of a class of standard 3' based arrays, ventromedial hypothalamic (VMH) lesions affected the expressions of cell proliferation-related genes, neuronrelated genes, and metabolism-related genes. The released Affymetrix Rat Gene 1.0 ST array has 2 major differences compared with standard $3^{\prime}$ based arrays, including Rat Genome 230 2.0: it interrogates the entire mRNA transcript and uses DNA targets. Purpose: This study is aimed at assessing the impact of these differences on the array performance. Methods: The study used Rat Gene 1.0 ST array, one of a class of whole-transcript rat exon arrays to examine the cellular mechanisms of gene regulation in the rat pancreas after VMH lesions. Results: Although the results showed that VMH lesions regulated genes involved in enzymes, metabolism, transport, binding differentiation, migration, morphology, apoptosis, neuron and immunity, the probes identified by these 2 arrays were remarkably different. Conclusion: This study also confirmed that $\mathrm{VMH}$ lesions may affect the expression of many functional genes in rat pancreas.
\end{abstract}

(c) 2017 S. Karger AG, Basel

\section{KARGER}

E-Mail karger@karger.com

www.karger.com/aon

\section{Introduction}

The pathways connecting the nervous system and gastrointestinal organs are a focus of attention among researchers [1]. Pancreas receives a rich supply of autonomic nerves [1]. The hypothalamus plays an important role in the consolidation of neurohumoral information, and has autonomic centers that are connected to the viscera through the autonomic nervous system [1]. Meanwhile, glucose increases the protein synthesis in $\beta$ cells overall and especially some secretory proteins, including insulin. Meanwhile, intracellular glucose signaling pathways control the secretion of glucose and insulin by pancreatic islet $\alpha$ - and $\beta$-cells, respectively [2]. However, glucose also indirectly controls the secretion of these hormones through the regulation of the autonomic nervous system, which richly innervates the endocrine organs. Both parasympathetic and sympathetic nervous systems also impact the endocrine pancreas postnatal development and plasticity in adult animals [2].

It was reported previously that ventromedial hypothalamic (VMH) lesions affected the expression of cell proliferation-related genes, neuron-related genes, and metabolism-related genes in a rat pancreas, using Rat Genome 2302.0 (Affymetrix Japan Co., Tokyo Japan) [3]. The released Affymetrix Rat Gene 1.0 ST array has 2 major differences compared with standard 3' based arrays, 
including Rat Genome 230 2.0: (i) it interrogates the entire mRNA transcript and (ii) it uses DNA targets. To assess the impact of these differences on the array performance, this study used Rat Gene 1.0 ST array, one of a class of whole-transcript rat exon arrays, to examine genes for which expression profiles showed significant transition. Moreover, the cellular mechanisms of gene regulation were investigated in the rat pancreas at day 3 after VMH lesions, because we previously reported that cell proliferation in the pancreas reaches a maximum at day $3[4]$.

\section{Methods}

\section{Animals}

Female Sprague-Dawley rats, weighing 230-250 g (age 1012 weeks), were used in this study. In VMH-lesioned rats, it was reported that the presence of a dark-light cycle had a profound influence on the postoperative feeding patterns, and there were marked sex differences where decreases in food consumption were seen more in female rats than in male rats within a day-night rhythmicity [5]. As such, female rats were used. They were maintained in a temperature- and light-controlled environment $(23 \pm$ $2{ }^{\circ} \mathrm{C} ; 12$-h light/12-h dark cycle) and were given free access to food and water. Tissue samples were collected from the pancreas of $\mathrm{VMH}-$ lesioned animals and sham-VMH-lesioned animals at day 3 after the operation ( $n=2$ in each group for DNA chips).

All animal procedures were performed in accordance with the Guidelines for Animal Experiments at the National Hospital Organization (NHO) Kure Medical Center and Chugoku Cancer Center. The protocols were approved by the Animals Research Ethics Committee, NHO Kure Medical Center and Chugoku Cancer Center.

\section{VMH Lesions}

VMH lesions or simulated operations were performed as previously described [6]. After the operations, the rats were returned to their cages and given free access to food and water. In this study and in all experiments, localization of the VMH lesions was confirmed by microscopic examination of the brain. Consistent with this finding, the previous report illustrates lesions that included the $\mathrm{VMH}[6]$.

\section{Total RNA Preparation and DNA Microarray Analysis}

In this study, the same sample using Rat Genome 2302.0 was not used, but the total RNA was isolated from fresh pancreatic tissue. We recently described a technique that reliably improves the amount and quality of RNA extracted from rat pancreas, an RNaserich organ, using RNAlater-ICE [7]. DNA microarray was performed as described previously [8]. In this study, double-stranded complementary DNA was comprised of hybridized Affymetrix GeneChip arrays (Rat Gene 1.0 ST Array; Affymetrix Japan Co., Tokyo, Japan). The digitized image data were processed using GeneChip Operating Software 1.4 (Affymetrix Japan Co.). Genes with fold-changes $>2.0$ were identified as differentially expressed genes. As replicate assays were not performed, a very stringent cut- off point was selected for the detection of significant upregulation or downregulation of genes in the mRNA amount between the arrays. Using the signal intensity of selected genes that were up- or downregulated compared with the sham-VMH-lesioned control group, analysis was performed using GeneSpring GX version 10 (Agilent Technologies, Santa Clara, CA, USA).

Biological Analysis of the Differential Expressed Gene Lists

Database for Annotation, Visualization, and Integrated Discovery (DAVID) were used for pathway analysis (http://david. abcc.ncifcrf.gov/), and provided lists of enriched KEGG pathways. A $p<0.05$ was set as the threshold.

\section{Results}

This study showed that VMH lesions regulated genes involved in enzymes, metabolism, transport, binding differentiation, migration, morphology, apoptosis, neuron, and immunity. Among 17,061 probes, the expression of 893 probes $(5.2 \%)$ was involved in at least a 2 -fold upregulation (324 probes) or downregulation (569 probes) at day 3 after $\mathrm{VMH}$ lesions as compared with sham-VMH lesions. Online supplementary Table 1 (see www.karger. com/doi/10.1159/000464420) shows the upregulated ( $>5$-fold) and downregulated ( $>5$-fold) genes, identified by Rat Gene 1.0 ST array. The results showed that VMH lesions regulated genes involved with enzymes (upregulated genes 3 probes; downregulated genes 5 probes), metabolism (upregulated genes 3 probes; downregulated genes 15 probes), transport (downregulated genes 2 probes), binding (downregulated genes 1 probe), differentiation (upregulated genes 2 probes; downregulated genes 2 probes), migration (upregulated genes 1 probe), morphology (upregulated genes 3 probes; downregulated genes 2 probes), apoptosis (downregulated genes 3 probes), neuron (downregulated genes 2 probes), and immune (upregulated genes 3 probes; downregulated genes 1 probe).

The results showed that VMH lesions upregulated genes related to inflammatory response, carbohydrate binding, response to hormone stimulus, response to endogenous, and response to extracellular stimulus, and downregulated genes related to immune response, response to nutrient levels, response to extracellular stimulus, response to hormone stimulus, and response to endogenous stimulus (Table 1). As shown in Table 1, some significantly enriched gene ontology (GO) terms were identified, and the most meaningful term was inflammatory response (GO 0006954, $p=8.4 \mathrm{E}-14)$ in upregulated gene families and immune response (GO 0006955, $p=$ 9.0E-11) in downregulated gene families. 
Table 1. The main functional enrichment results of the interaction network in upregulated ( $>2$-fold) and downregulated ( $>2$-fold) genes in pancreatic islets after $\mathrm{VMH}$ lesioning

\begin{tabular}{|c|c|c|c|}
\hline GO term & Description & Genes & $p$ value \\
\hline $\begin{array}{l}\text { Upregulated } \\
0006954\end{array}$ & Inflammatory response & $\begin{array}{l}\text { Cd14, Cd44, Fcgr2b, Alox5, Ccl2, Ccl7, Ccr1, Ccr2, C1qc, C1qa, } \\
\text { C1qb, C2, C3, C6, Cfd, Cfh, Cfp, Fn1, Itgb2, Lipa, Ptafr, Reg3a, Reg3b, } \\
\text { Serpina3n, Slc11a1, S1pr3, Tfrc, Vnn1, Vcam1 }\end{array}$ & $8.4 \mathrm{E}-14$ \\
\hline 0030246 & Carbohydrate binding & $\begin{array}{l}\text { Adamts1, Clec4a1, Clec4a3, Clec4d, Clec7a, Cd44, Aldob, Ccl2, } \\
\text { Ccl7, Ccdc80, Cfh, Fn1, Fstl1, Gys2, Gpnmb, Itgam, Lgals1, Lgals3, Lpl, } \\
\text { Lyve1, Clec10a, Mrc1, Postn, Prelp, Prg4, Reg3a, Reg3b, Vcan }\end{array}$ & $8.0 \mathrm{E}-13$ \\
\hline 0009725 & $\begin{array}{l}\text { Response to hormone } \\
\text { stimulus }\end{array}$ & $\begin{array}{l}\text { Cd38, Timp1, Adipoq, Aldob, Anxa1, Apobec1, Cav1, Ccl2, Ccr2, } \\
\text { Col1a1, C1qb, Cdkn1a, Cdo1, Dusp1, Fabp4, Gpx3, Ldha, Lcat, Lep, } \\
\text { Lpin1, Ldlr, Lox, Me1, Oxtr, Pparg, Pla2g4a, Prkar2b, Retn, Rbp4, } \\
\text { Sparc, Serpina3n, Serpinf1, Insig1, Cd36, Tnfrsf11b }\end{array}$ & $7.3 \mathrm{E}-12$ \\
\hline 0009719 & $\begin{array}{l}\text { Response to endogenous } \\
\text { stimulus }\end{array}$ & $\begin{array}{l}\text { Cd38, Timp1, Adipoq, Aldob, Anxa1, Apobec1, Cav1, Ccl2, Ccr2, } \\
\text { Col1a1, C1qb, Cdkn1a, Cdo1, Dusp1, Fabp4, Gpx3, Ldha, Lcat, Lep, } \\
\text { Lpin1, Ldlr, Lox, Me1, Mgst1, Oxtr, Pparg, Pla2g4a, Prkar2b, Retn, } \\
\text { Rbp4, Sparc, Serpina3n, Serpinf1, Insig1, Cd36, Tnfrsf11b }\end{array}$ & $4.0 \mathrm{E}-11$ \\
\hline $\begin{array}{l}\text { Downregulated } \\
0006955\end{array}$ & Immune response & $\begin{array}{l}\text { Cd4, Cd74, Fcer1g, Fcgr2b, RT1-Ba, Sp100, Apobec1, B2m, Ccl2, } \\
\text { Ccl21b, Ccl24, Ccl6, Ccr2, Cxcl13, Cxcl9, Colec12, C1qa, C1qb, C2, } \\
\text { C6, Cfd, Cfp, Cybb, Gbp2, Gbp5, RT1-Da, Il1rl1, RT1-DMb, Ptprc, } \\
\text { Rsad2, Serping1, C1s, Tlr7, Tgfbr3, Tnfsf10, Vnn1 }\end{array}$ & $9.0 \mathrm{E}-11$ \\
\hline 0031667 & $\begin{array}{l}\text { Response to nutrient } \\
\text { levels }\end{array}$ & $\begin{array}{l}\text { Pfkfb1, Abca1, Cd38, H19, Acsl1, Adipoq, Alb, Aldh1a1, Aldh1a2, } \\
\text { Alox5, Cav1, Cp, Ccl2, Ccr2, Col1a1, C2, Ccnd1, Cybb, Lep, Npy, } \\
\text { Pparg, Rbp4, Sparc, Serpinf1, Cd36, Tgfbr2, Ucn3, Vcam1 }\end{array}$ & $9.7 \mathrm{E}-10$ \\
\hline 0009719 & $\begin{array}{l}\text { Response to endogenous } \\
\text { stimulus }\end{array}$ & $\begin{array}{l}\text { Pfkfb1, Cd38, H19, Timp1, Adipoq, Aldh1a1, Aldh1a2, Anxa1, Apobec1, } \\
\text { Aqp1, Cav1, Ccl2, Ccr2, Col1a1, C1qb, Ccnd1, Cdo1, Esr1, Fabp4, Gstm1, } \\
\text { Gpx3, Lep, Lox, Me1, Mgst1, Oxtr, Pparg, Pde3b, Pck1, Plat, Prkar2b, } \\
\text { Rbp4, Sparc, Serpina3n, Serpinf1, Cd36, Tgfbr2, Tgfbr3, Tnfsf10, Ucn3 }\end{array}$ & $4.1 \mathrm{E}-9$ \\
\hline
\end{tabular}

DAVID functional annotation analysis showed that the molecular pathways enriched in upregulated gene families included those associated with complement and coagulation cascades, circadian rhythm, PPAR signaling pathway, hematopoietic cell lineage, systemic lupus erythematosus, pyruvate metabolism, ether lipid metabolism, glycerophospholipid metabolism, renin-angiotensin system, arachidonic acid metabolism, and neuroactive ligand receptor-interaction, whereas those in downregulated gene families included complement and coagulation cascades, the PPAR signaling pathway, systemic lupus erythematosus, ECM-receptor interaction, ether lipid metabolism, renin-angiotensin system, focal adhesion, cell adhesion molecules, glycerophospholipid metabolism, arachidonic acid metabolism, hematopoietic cell lineage, retinol metabolism, cytokine-cytokine receptor interaction, antigen proceeding and presentation, and the chemokine signaling pathway (Table 2). 
Table 2. Enriched pathways in upregulated ( $>2$-fold) and downregulated ( $>2$-fold) genes identified by DNA microarray analysis at 3 days in pancreatic islets after VMH lesioning

\begin{tabular}{|c|c|c|c|c|c|}
\hline Category & Term & Count & $\%$ & $p$ value & Benjamini \\
\hline \multicolumn{6}{|l|}{ Upregulated } \\
\hline KEGG_PATHWAY & Complement and coagulation cascades & 13 & 4.6 & $6.0 \mathrm{E}-8$ & $6.5 \mathrm{E}-6$ \\
\hline KEGG_PATHWAY & Circadian rhythm & 6 & 2.1 & $7.5 \mathrm{E}-6$ & $4.1 \mathrm{E}-4$ \\
\hline KEGG_PATHWAY & PPAR signaling pathway & 10 & 3.5 & $3.7 \mathrm{E}-5$ & $1.3 \mathrm{E}-3$ \\
\hline KEGG_PATHWAY & Hematopoietic cell lineage & 9 & 3.2 & $4.6 \mathrm{E}-4$ & $1.2 \mathrm{E}-2$ \\
\hline KEGG_PATHWAY & Systemic lupus erythematosus & 9 & 3.2 & $1.2 \mathrm{E}-3$ & $2.5 \mathrm{E}-2$ \\
\hline KEGG_PATHWAY & Pyruvate metabolism & 5 & 1.8 & $1.3 \mathrm{E}-2$ & $2.1 \mathrm{E}-1$ \\
\hline KEGG_PATHWAY & Ether lipid metabolism & 4 & 1.4 & $4.5 \mathrm{E}-2$ & $5.1 \mathrm{E}-1$ \\
\hline KEGG_PATHWAY & Glycerophospholipid metabolism & 5 & 1.8 & $5.8 \mathrm{E}-2$ & $5.1 \mathrm{E}-1$ \\
\hline KEGG_PATHWAY & Renin-angiotensin system & 3 & 1.1 & $8.0 \mathrm{E}-2$ & $5.9 \mathrm{E}-1$ \\
\hline KEGG_PATHWAY & Arachidonic acid metabolism & 11 & 3.9 & $9.0 \mathrm{E}-2$ & $5.7 \mathrm{E}-1$ \\
\hline KEGG_PATHWAY & Neuroactive ligand receptor-interaction & 5 & 0.9 & $7.2 \mathrm{E}-3$ & $4.9 \mathrm{E}-2$ \\
\hline \multicolumn{6}{|l|}{ Downregulated } \\
\hline KEGG_PATHWAY & Complement and coagulation cascades & 11 & 2.8 & $1.6 \mathrm{E}-5$ & $1.9 \mathrm{E}-3$ \\
\hline KEGG_PATHWAY & PPAR signaling pathway & 11 & 2.8 & $1.8 \mathrm{E}-5$ & $1.1 \mathrm{E}-3$ \\
\hline KEGG_PATHWAY & Systemic lupus erythematosus & 12 & 3.0 & $2.7 \mathrm{E}-5$ & $1.1 \mathrm{E}-3$ \\
\hline KEGG_PATHWAY & ECM-receptor interaction & 8 & 2.0 & $6.2 \mathrm{E}-3$ & $1.7 \mathrm{E}-1$ \\
\hline KEGG_PATHWAY & Ether lipid metabolism & 5 & 1.3 & $1.3 \mathrm{E}-2$ & $2.6 \mathrm{E}-1$ \\
\hline KEGG_PATHWAY & Renin-angiotensin system & 4 & 1.0 & $1.6 \mathrm{E}-2$ & $2.5 \mathrm{E}-1$ \\
\hline KEGG_PATHWAY & Focal adhesion & 12 & 3.0 & $1.7 \mathrm{E}-2$ & $1.3 \mathrm{E}-3$ \\
\hline KEGG_PATHWAY & Cell adhesion molecules (CAMs) & 10 & 2.5 & $1.9 \mathrm{E}-2$ & $2.5 \mathrm{E}-1$ \\
\hline KEGG_PATHWAY & Glycerophospholipid metabolism & 6 & 1.5 & $2.6 \mathrm{E}-2$ & $2.7 \mathrm{E}-1$ \\
\hline KEGG_PATHWAY & Arachidonic acid metabolism & 6 & 1.5 & $4.1 \mathrm{E}-2$ & $3.4 \mathrm{E}-1$ \\
\hline KEGG_PATHWAY & Hematopoietic cell lineage & 6 & 1.5 & $6.0 \mathrm{E}-2$ & $4.8 \mathrm{E}-1$ \\
\hline KEGG_PATHWAY & Retinol metabolism & 5 & 1.3 & $7.6 \mathrm{E}-2$ & $4.8 \mathrm{E}-1$ \\
\hline KEGG_PATHWAY & Cytokine-cytokine receptor interaction & 10 & 2.5 & $8.7 \mathrm{E}-2$ & $5.1 \mathrm{E}-1$ \\
\hline KEGG_PATHWAY & Antigen proceeding and presentation & 6 & 1.5 & $9.1 \mathrm{E}-2$ & $5.0 \mathrm{E}-1$ \\
\hline KEGG_PATHWAY & Chemokine signaling pathway & 9 & 2.3 & $9.3 \mathrm{E}-2$ & $4.9 \mathrm{E}-1$ \\
\hline
\end{tabular}

In a previous study, we described upregulated $(>3$-fold) genes and downregulated ( $>3$-fold) genes, identified by the Genome 2302.0 array. Table 3 shows a comparison of categories in upregulated ( $>3$-fold) and downregulated (>3-fold) genes identified by the Genome 2302.0 array and Gene 1.0 ST array at 3 days in pancreatic islets after VMH lesions. Genome 2302.0 array identified $13(0.4 \%)$ upregulated probes and 23 (0.7\%) downregulated probes among 31,099 probes; meanwhile, Gene 1.0 ST array identified 70 (4.1\%) upregulated probes and 117 (6.9\%) downregulated probes among 17,061 probes. In the Genome 2302.0 array, the common categories in upregulated genes were enzyme (31\%), migration (24\%), and morphology $(15 \%)$, and those in downregulated genes were enzyme (43\%), differentiation (17\%), morphology (17\%), and neuron (17\%). In comparison, in the Gene $1.0 \mathrm{ST}$ array, the common categories in upregulated genes were immune (19\%), metabolism (17\%), and morphology (16\%), and those in downregulated genes were metabolism (23\%), immune (19\%), differentiation (10\%), and neuron (10\%) (Table 3). Consistent with this, we recently reported that using Gene 1.0 ST array in rat pancreatic islets rather than whole pancreatic tissues, VMH lesions affect the expressions of multiple cell proliferation, morphology, metabolism, and immune response genes [8-10]. Among the probes identified by these arrays, in downregulated genes, the same probes consisted of only 2 probes: L-threonine dehydrogenase and murinoglobulin 1, but in upregulated genes, the same probes were not found.

\section{Discussion}

Current publication guidelines require that all microarray results are confirmed by an independent gene expression profiling method, such as real-time PCR, because microarrays can also give results that vary considerably [11]. However, it is impossible to confirm all 
Table 3. Comparisons of categories in upregulated ( $>3$-fold) and downregulated (>3-fold) genes identified by Genome 2302.0 array, one of standard $3^{\prime}$ based arrays and Gene 1.0 ST array, one of whole-transcript rat exon arrays, at 3 days in pancreatic islets after VMH lesioning

\begin{tabular}{|c|c|c|}
\hline Category & Genome $2302.0, n(\%)$ & Gene 1.0 ST array, $n(\%)$ \\
\hline \multicolumn{3}{|l|}{ Upregulated } \\
\hline Apoptosis & 0 & $2(3)$ \\
\hline Binding & $1(8)$ & $1(1)$ \\
\hline Circadian & 0 & $2(3)$ \\
\hline Differentiation & $1(8)$ & $7(10)$ \\
\hline Enzyme & $4(31)$ & $6(9)$ \\
\hline Growth & 0 & $2(3)$ \\
\hline Hormone & $1(8)$ & $2(3)$ \\
\hline Immune & 0 & $13(19)$ \\
\hline Metabolism & 0 & $12(17)$ \\
\hline Migration & $3(24)$ & $2(3)$ \\
\hline Morphology & $2(15)$ & $11(16)$ \\
\hline Neuron & $1(8)$ & $1(1)$ \\
\hline Signaling & 0 & $6(9)$ \\
\hline Transport & 0 & $3(4)$ \\
\hline Total & 13 & 70 \\
\hline \multicolumn{3}{|l|}{ Downregulated } \\
\hline Apoptosis & 0 & $6(5)$ \\
\hline Binding & $1(4)$ & 0 \\
\hline Coagulation & 0 & $1(1)$ \\
\hline Differentiation & $4(17)$ & $12(10)$ \\
\hline Enzyme & $10(43)$ & $7(6)$ \\
\hline Growth & 0 & $2(2)$ \\
\hline Hormone & 0 & $4(3)$ \\
\hline Immune & 0 & $22(19)$ \\
\hline Metabolism & 0 & $27(23)$ \\
\hline Migration & 0 & $6(5)$ \\
\hline Morphology & $4(17)$ & $12(10)$ \\
\hline Neuron & $4(17)$ & $10(9)$ \\
\hline Signaling & 0 & $3(3)$ \\
\hline Transport & 0 & $5(4)$ \\
\hline Total & 23 & 117 \\
\hline
\end{tabular}

microarray results by real-time PCR because of the high data volumes and often large genomes.

In this study, Rat Gene 1.0 ST array was used, which is one of a class of whole-transcript rat exon array for mRNA expression profiling of rat pancreas, to examine pancreatic cellular responses to $\mathrm{VMH}$ lesions, and showed the fact that $\mathrm{VMH}$ lesions regulated genes were involved in many functions related to the pancreas, including for enzymes, metabolism, transport, binding, differentiation, migration, morphology, apoptosis, neuron, and immune function. We previously reported that using Genome 2302.0 , one of a class of standard $3^{\prime}$ based arrays, VMH lesions influenced genes that were involved in functions related to cellular compromise, immune response, immune and lymphatic system development and function, cancer, hematological disease, cellular movement, molecular transport, cell signaling, nerve system development and function, etc., in whole pancreatic tissue [3]. Therefore, our previous results [3] were confirmed in this study, using whole-transcript rat exon array, because VMH lesions change the expression of many function genes in rat pancreas. However, the probes identified by these 2 arrays were remarkably different (Table 3 ). Further studies are needed to clarify this finding.

In this study, we examined freshly isolated islets for gene expression analysis. Islet isolation shows the change of expression of multiple genes including those involved in hypoxia and stress [12]. However, functional cluster analysis did not indicate an enrichment of genes in these categories. Therefore, there is almost no possibility that islet isolation itself may alter the gene expressions in the present study.

In conclusion, many genes according to $\mathrm{VMH}$ lesions were also identified by whole-transcript rat exon array. These genes may effect changes in the pancreas, both due to VMH-specific effects and secondary effects of metabolic consequences from VMH lesion. This study showed multiple genes and gene clusters that might clarify additional gene functions that affect pancreas functions independently or in combination with VMH lesions. Recently, while it is true that qPCR cannot validate every single gene that is up- or downregulated, it can now come closer with the advent of Fluidigm and related technologies. Further investigations may be needed to clarify the validation by these technologies. One of the limitations of this study is that 2 array systems were not compared with the same RNA samples. In this experiment, the study designs have similar components regarding the age of mice, housing conditions, differences, and severity of VMH lesions. However, in case of fragile tissues like islets, slight variations in glucose concentrations, oxygen, and stress may influence a variety of genes.

\section{Acknowledgements}

This work was supported in part by grants-in-aid from grant 26461042 from the Ministry of Education, Culture, Sports, Science and Technology in Japan. The funder had no role in study design, data collection and analysis, decision to publish, or preparation of the manuscript.

\section{Disclosure Statement}

The author declares no conflict of interest. The manuscript is complied with International Committee of Medical Journal Editor's guidelines. 


\section{References}

1 Kiba T: The role of the autonomic nervous system in liver regeneration and apoptosis recent developments. Digestion 2002;66:7988.

2 Thorens B: Neural regulation of pancreatic islet cell mass and function. Diabetes Obes Metab 2014;16(suppl 1):87-95.

3 Kiba T, Kintaka Y, Suzuki Y, Ishizuka N, Ishigaki Y, Inoue S: Gene expression profiling in rat pancreas after ventromedial hypothalamic lesioning. Pancreas 2010;39:627-632.

4 Kiba T, Tanaka K, Numata K, Hoshino M, Misugi K, Inoue S: Ventromedial hypothalamic lesion-induced vagal hyperactivity stimulates rat pancreatic cell proliferation. Gastroenterology 1996;110:885-893.

5 Balagura S, Devenport LD: Feeding patterns of normal and ventromedial hypothalamic le- sioned male and female rats. J Comp Physiol Psychol 1970;71:357-364.

6 Kiba T, Tanaka K, Endo O, Inoue S: Ventromedial hypothalamic lesions increase gastrointestinal DNA synthesis through vagus nerve in rats. Gastroenterology 1993;104: 475-484.

7 Kiba T, Kintaka Y, Nakada E, Suzuki Y, Inoue S, Ishigaki Y: High-quality RNA extraction from rat pancreas for microarray analysis. Pancreas 2007;35:98-100.

8 Kiba T, Ishigaki Y: Ventromedial hypothalamic lesions change the expression of cell proliferation-related genes and morphologyrelated genes in rat pancreatic islets. Islets 2014;6:e1012950.

9 Kiba T: Changes of the expressions of multiple metabolism genes in rat pancreatic islets after ventromedial hypothalamic lesioning. Neurosci Lett 2015;604:64-68.

10 Kiba T: Ventromedial hypothalamic lesions downregulate multiple immune signaling pathways in rat pancreatic islets. Neurosci Lett 2016;610:177-181.

11 Bosotti R, Locatelli G, Healy S, Scacheri E, Sartori L, Mercurio C, Calogero R, Isacchi A: Cross platform microarray analysis for robust identification of differentially expressed genes. BMC Bioinformatics 2007;8(suppl 1): S5.

12 Marselli L, Thorne J, Ahn YB, Omer A, Sgroi DC, Libermann T, Otu HH, Sharma A, Bonner-Weir S, Weir GC: Gene expression of purified beta-cell tissue obtained from human pancreas with laser capture microdissection. J Clin Endocrinol Metab 2008;93:1046-1053. 